\title{
El turismo, pilar del desarrollo local. Los paladares en Cuba.
}

Tourism, pillar of local development. The palates in Cuba.

Dra.C Lourdes Cisneros Mustelier. ${ }^{1}$ Lic. Diana Llanes Rosales. ${ }^{2}$.

\begin{abstract}
The objective of this work is to demonstrate the need to promote the creation of local projects that revalue traditions, customs, habits and history. With the launch of this entrepreneurial municipal initiative, the multiplier effect of Tourism on the rest of the sectors of the territory would be highlighted, achieving great benefits with growing social and economic development for the population.

In the research, theoretical level methods such as historical-logical, hypothetical deductive and others such as statistical and mathematical were used. In addition, sources of information and research techniques were used such as: interviews with owners and workers of non-state businesses, observation, bibliographic review among others that contributed to the analysis of aspects related to tourism, gastronomy and non-state establishments. state restoration.
\end{abstract}

Key Words: Gastronomic tourism; Local development; Cuban cuisine; Non-state establishments..

\section{Resumen}

El objetivo del presente trabajo radica en demostrar la necesidad de impulsar la creación de proyectos locales que revaloricen las tradiciones, costumbres, hábitos e historia. Con la puesta en marcha de esta iniciativa municipal emprendedora, se resaltaría el efecto multiplicador del Turismo sobre el resto de los sectores del territorio, lográndose grandes beneficios con un creciente desarrollo social y económico para la población. En la investigación se emplearon los métodos de nivel teórico como el histórico-lógico, el hipotético deductivo y otros como el estadístico y matemático. Además, se emplearon

\footnotetext{
${ }^{1}$ Prof. Auxiliar, Facultad de Turismo, Universidad de la Habana, lourdes_cisneros@ftur.uh.cu

${ }^{2}$ Facultad de Turismo, Universidad de la Habana, dianallanes1995@gmail.com
} 
fuentes de información y técnicas de investigación tales como: entrevistas a propietarios y trabajadores de negocios no estatales, la observación, la revisión bibliográfica entre otras que tributaron al análisis de los aspectos relacionados con la actividad turística, la gastronomía y los establecimientos no estatales de restauración.

Palabras Clave: Turismo Gastronómico; Desarrollo local; Cocina cubana; Establecimientos no estatales.

\section{Introducción}

Mundialmente todos los países que desarrollan la industria del turismo buscan ofrecer al mundo su cultura culinaria y todos poseen historias gastronómicas únicas; plagadas de olores, sabores y colores que son de gran agrado a los clientes que lo reciben. Los visitantes internacionales buscan conocer esa cultura, pero los nativos adoran su propia comida y la prefieren consumir cuando tienen posibilidades de incursionar en el mundo del turismo.

Convertir la actividad turística en factor determinante para la nación, desde la comunidad es razón suficiente para que el desarrollo turístico y el desarrollo local vayan de la mano, para esto se hace necesario ordenar adecuadamente los atractivos del municipio para que tengan un valor significativo como destino turístico y por lo tanto, se constituya como elemento sustancial del desarrollo económico de la localidad, además pueda convertirse en un producto competitivo, sin riesgo de desaparecer con el tiempo.

En el caso de Cuba, el desarrollo local se enfoca más como: “el proceso mediante el cual esa escala implementa las necesarias transformaciones en las dimensiones: ambientaleconómico-productiva y político-social, a partir de una proyección estratégica elaborada y un plan que se cambiará y evolucionará con la práctica de los propios gestores. Pero este desarrollo no es totalmente independiente, sino que debe mantenerse interconectado con el entorno y formando parte del desarrollo nacional" (Guzón, 2003).

Aún así persisten trabas que imposibilitan el desarrollo local en comunidades que tienen potencialidades para su desarrollo, entre ellas la carencia de instrumentos en la escala local para la planificación y gestión de actividades turísticas y la complejidad que entraña un sistema de este tipo: Para eliminar estos obstáculos, Cuba pretende enfrentar el nuevo reto con un cambio de mentalidad en los actores; así como elaborando ofertas locales que integren los valores del territorio, su gente, sus costumbres, tradiciones e identidad, que los diferencie para insertarse en un mundo turístico globalizado, especializado y de calidad.

El mayor de estos desafíos está en que nuestro país tiene que asumir desde su cultura, desde su localidad, lo diferente, lo participativo, lo social, lo medioambiental, y desde lo 
económico la integración de todos los actores, incluyendo la comunidad, sus habitantes y sus espacios para imponerse como destino turístico preferencial (Betancourt, 2012).

Desde los años 90 y hasta la actualidad las formas de gestión no estatal, que con más fuerza y rapidez proliferaron en Cuba, fueron los asociados al alojamiento y la restauración, y la mayoría operaron en sus inicios con el turismo internacional como mercado meta.

\section{Desarrollo}

\section{La cocina cubana un punto y aparte}

"La cocina es una forma universal de la actividad humana, tal como no es posible la existencia de una sociedad sin lenguaje, tampoco existe ninguna que no cocine de uno u otro modo determinados alimentos, convirtiéndose en una especie de lenguaje al que se le traduce sus estructuras inconscientes y en el cual intenta resolver y revelar determinadas contradicciones.

Entonces nos toca a nosotros los cubanos escoger nuestro "estilo étnico" y, en medio de tantos cambios, limitaciones y reticencias, decidir cómo hacemos para "traducir nuestras estructuras inconscientes", según disciernen los citados estudiosos de las ciencias sociales, "el lenguaje simbólico de las comidas", tratando de acostumbrar el criollismo del paladar a estos nuevos sabores, posibilidades y costumbres en los cuales hemos quedado inmersos, así como ocuparnos de la sustitución de aquella memorias gustativas a las cuales ya no tenemos acceso, mientras intentamos "solventar y revelar" nuestras propias contradicciones, "traduciéndolas", no según dictan nuestros gustos y costumbres, sino en función de las delimitadas posibilidades simbólicas de la cocina, alimentos y sabores, que nos permiten hoy nuestra época y alcance económico" (Dordevic, 2006).

La mayor de la Antillas tiene una forma de cocinar especial, esta tipicidad se le atribuye a la confluencia de dos factores, el español y el africano, aunque en épocas posteriores se sumaron elementos de la comida asiática, con la emigración china a finales del siglo XIX, incorporándose además lo norteamericano, lo yucateco, lo haitiano, lo jamaicano y lo caribeño en general; e incluso con reminiscencia de lo árabe y lo judío. ¡Todo un ajiaco! ... que, con el filtro de los siglos desembocó en un producto nuevo y autóctono: lo cubano.

A contrapelo con la opinión de algunos sobre la sencillez y pocas recetas, el arte culinario cubano constituye un libro abierto de nuevas páginas que se revitalizan día a día, como lo reafirman muchos expertos en el tema, mezclando lo suculento, lo delicado, lo atrevido, lo creativo y lo relevante que puede extraerse de los productos y del recetario cubano, hijo de la constante mezcla que somos, para así lograr mayor adaptación y creatividad, viviendo así de nuestra cocina, pero redirigiendo parte de ella hacia los códigos actuales. 
A su vez, hay que ir educando los sentidos para percibir los detalles, lo exquisito, para que lo excepcional, de lo tradicional, navegue cargado de cuanta avidez se logre por lo estético, inteligente, delicado y ético del comer y el beber. Hay que reinterpretar y rediseñar la cocina de antaño, los productos de siempre junto a los nuevos y actuales, sin olvidar que para esto debemos entrenar los sentidos, culturizar la estética, educar la sensibilidad, entender el fenómeno de lo simple a lo complejo y viceversa. Lo hibrido hace parte de nuestras vidas, dando lugar a una fusión interesantísima. La mezcla es la clave del éxito porque incluye lo transcultural, mostrando lo diverso. Hoy se impone la identidad, pero como mezcla de lo gourmet y lo tradicional. El futuro de la gastronomía, sin dejar de ser el amasijo cultural que somos, dispares en esencia y apariencia, será del arte intuitivo culinario (Loyola, 2014).

\section{Origen y evolución de los establecimientos no estatales de restauración gastronómica en Cuba (paladares).}

Un antecedente poco mencionado de los negocios no estatales de restauración gastronómica proviene del movimiento "underground dining " o "clandestine dining" que incluyen conceptos tan atractivos como los restaurantes clandestinos, llamados también suppers clubs, los pop-up restaurants o los restaurantes en casas particulares, garajes, tiendas o incluso "salas secretas" dentro de un restaurante, que pasan desapercibidos para la mayoría de las personas y cuyo gancho es precisamente su exclusividad y diferencia frente a los formatos convencionales. Que estos establecimientos sean clandestinos no significan que sean ilegales, se mueven en la clandestinidad simplemente con el objetivo de fomentar la exclusividad y el misterio dentro de la clientela. Además, cuentan con su licencia, sus permisos, perfil en Facebook y redes sociales.

El origen de este concepto podemos encontrarlo en antros de copas y comidas de la" Ley Seca" en los Estados Unidos, los paladares cubanos o las casas restaurantes de Chefs en Buenos Aires. La necesidad de dar una oferta prohibida en el caso de la Ley Seca y la necesidad de sobrevivir económicamente en tiempos difíciles como en Cuba y Argentina fueron los motores impulsores para esta actividad. "El Paladar Cubano" es el formato que más se asemeja con la tendencia actual.

Paladar es un término empleado exclusivamente en Cuba para designar a restaurantes montados y dirigidos por cuentapropistas. El término, de uso popular, tiene su origen en la telenovela brasileña Vale Todo, mostrada en Cuba a inicios de la década de los años 90. Paladar era el nombre de la cadena de restaurantes dirigidos por Raquel Accioli, la protagonista, interpretada por Regina Duarte. La transmisión de esta telenovela coincidió en la época que se concedieron las primeras licencias para el trabajo cuentapropista en Cuba, por lo que el público cubano bautizó así a los entonces establecimientos de nuevo tipo. 
Precediendo estos acontecimientos la Gaceta Oficial de la República de Cuba publica el día 8 de octubre de 2010 una serie de actividades autorizadas para el ejercicio por cuenta propia, donde aparece relacionado el ejercicio de "elaborador/ vendedor de alimentos y bebidas mediante servicio gastronómico, ejerciendo la actividad en su domicilio mediante el uso de mesas, sillas, banquetas o similares hasta 20 capacidades.

Posteriormente, en diciembre de 2010 y hasta febrero de 2011 se desarrolla un proceso de discusión del proyecto de Lineamientos de la Política Económica y Social del Partido y la Revolución, referidos en el Capítulo I, donde como constancia de la primera etapa de implementación de estas medidas es derogada la Resolución anterior y toma papel legal la Resolución 33/2011 de la Ministra de Trabajo y Seguridad Social que otorga un mayor número de capacidades en el ejercicio del trabajo por cuenta propia, esta vez posibilitando el soporte de servicio para 50 comensales.

\section{Protagonismo de los negocios no estatales de restauración gastronómica dentro del sector turístico.}

Cuando triunfa la revolución, en el ámbito cubano ya existía una tradición multinacional en la cocina cubana, no precisamente con fines turísticos, sino satisfaciendo los gustos de una población donde coexisten una mezcla de identidades culturales, representadas en la existencia, desde las humildes fondas y trenes de cantina, hasta encumbrados restaurantes especializados en comidas internacionales.

Esto hace que la actividad turística en Cuba alcance un crecimiento acelerado que ha permitido dinamizar la economía sobre la base de un programa de desarrollo eficiente, incidiendo en ello el mejoramiento de la oferta gastronómica, destacándose la actividad no estatal gastronómica, la cual se ha ido posicionando dentro de la competencia con las empresas extra hoteleras.

Dan fe de esto las opiniones de los clientes que acceden a sitios de intercambio de información y a la propaganda de las redes sociales. Hoy no pocas de estas instalaciones están insertadas en guías alternativas para el turismo internacional, porque son más pequeñas, que los grandes hoteles, y brindan una atención más personalizada. De ahí que la elaboración y venta de alimentos, sea una de las actividades más emprendedoras por el sector no estatal cubano y ocupan el segundo lugar en el trabajo por cuenta propia, justo detrás de los transportistas privados.

\section{Caracterización de los establecimientos no estatales de restauración gastronómica (paladares) en La Habana.}

Desde el surgimiento de la actividad de restauración en el sector no estatal, puede plantearse que existen una serie de parámetros que influyen de modo permanente en el desarrollo de la misma. La capacidad de estos establecimientos es considerable, hasta 50 
comensales, pero marca cierto grado de limitación si se hace referencia a la imposibilidad de poder realizar actividades de medio formato para festividades, como cumpleaños, bodas, entre otros motivos. Es una arista que recurre a una de las tantas desventajas o perfiles que pudieran hacer menos atractiva a un paladar.

En cierta medida estos negocios cuentan con excelentes trabajadores, muchos de ellos con experiencia en el sector del turismo, otros son personas que han adquirido conocimientos empíricamente pero con pleno dominio del oficio que realizan, y específicamente en el servicio gastronómico la fuerza de trabajo es un personal joven que proviene del sector universitario y laboran en el horario no escolar en estos establecimientos, ellos quizás no tengan la experiencia necesaria y en ocasiones puede ser un personal itinerante debido a la intensidad del trabajo que genera la actividad gastronómica pero son ágiles en el trabajo y con mucha disposición para asimilar todas las actividades que se les asignen.

Desde el punto de vista organizativo estos centros no están integrados por departamentos que permitan controlar y viabilizar su gestión de una manera más efectiva tanto de modo directo o de apoyo a los servicios. A tal situación es preciso establecer que la mayoría de estas acciones generalmente recaen en los hombros de los dueños de estos negocios, quienes deben contabilizar un estado relativamente suntuoso de operaciones, generalmente de forma manual y sin disponer, en muchos casos, del conocimiento necesario para ello.

Un ejemplo claro de lo descrito anteriormente es apreciable en las operaciones correspondientes a las partidas de ingresos, costos y gastos. Estos procesos no se llevan a cabo de una manera planificada, pues no se fijan metas o se estima algún presupuesto. No existe una noción clara de la importancia de contar con ciertos medios que minimizan obstáculos en estos procesos, tales como las conocidas fichas técnicas y los escandallos de producción. Procesos como el de compra e inventarios no están sujetos a la aplicación de algún modelo o sistema, simplemente se ejecutan acorde a las necesidades diarias.

En torno a esta disyuntiva debe subrayarse que estos restaurantes han sido situados en locales adaptados y no concebidos para esta funcionalidad, por lo que en muchos casos no cuentan con las condiciones óptimas para el almacenamiento de diversas materias primas.

Otro aspecto que las caracterizan es que en general, no cuentan con sistemas de gestión de la calidad incorporada y que suelen desconocerse algunos procesos cruciales en el desempeño de la actividad por parte del cliente interno; es indiscutible que la calidad es concebida y asumida como una necesidad primaria en el negocio. Es fácil atestiguarlo al visitar la mayoría de estos sitios y percatarse en el esmero y el sentido de pertenencia con que se labora, siempre se piensa en los resultados y en las mejoras que han de implementarse. 
Situación actual de los paladares en la habana. Estudio de caso: municipio centro Habana.

La existencia de estos restaurantes privados especializados en las más diversas tradiciones culinarias nacionales e internacionales, sitúa parte de la actividad gastronómica local entre las nuevas formas de gestión no estatal con mayor dinamismo y originalidad del territorio. Esto resulta muy favorable porque, primero una buena gastronomía es tarea pendiente desde hace mucho tiempo; segundo, de tantos "timbiriches" y restaurantes que se han levantado en una y otra esquina, pocos estaban a la altura, ni tan siquiera clasificaban para segunda categoría. Tercero y, por último, en muchos de estos espacios sociales, que lo son, van de la mano profesionalidad, compromiso con el cliente y pensamiento en grande, lo cual se traduce simplemente, en hacer bien las cosas, intentar ser los mejores e innovar todo el tiempo.

Problemática Vigente:

- Dificultades en la posibilidad de acceder de manera estable a determinados suministros.

- Falta de estabilidad para adquirir insumos y altos precios que se pagan por las copas, vajillas, vasos, cubertería al no existir un mercado donde comprar más barato.

- La ausencia de un mercado mayorista estable de materias primas, es uno de los principales inconvenientes que inciden de forma directa.

- La adquisición de los recursos necesarios en el mercado negro, lo cual repercute en el precio de los productos, altera la estabilidad de los costos de elaboración, distorsiona las partidas de gastos reales justificables e incentiva las ilegalidades.

- Limitaciones en cuanto a la obtención de créditos bancarios y financiamiento de inversiones.

- Dificultades para el remozamiento y mantenimiento de los espacios que ocupan estos establecimientos que generalmente son viviendas muy antiguas y necesitan una inversión preliminar a veces elevada para garantizar su acondicionamiento.

- Retos y desafíos:

- Estimular la creatividad y la competitividad.

- Mejorar la economía individual y colectiva.

- Aumentar y fomentar la capacitación, la educación y la formación.

- Lograr la fusión cultura-turismo.

- Aumentar la calidad de las propuestas gastronómicas y posicionarse como atractivo turístico local.

- Incorporar visitantes que viajen motivados por la gastronomía.

- Comprender mejor el fenómeno del turismo gastronómico para demostrar que la gastronomía puede ser un atractivo turístico primario. 
- Identificar las principales motivaciones gastronómicas y distinguir si las personas que viajan por motivos gastronómicos asocian la gastronomía a la cultura de la localidad.

Evaluación de las Cinco Fuerzas de Porter para los restaurantes no estatales.

1-Intensidad de la rivalidad: Gran parte de los establecimientos no estatales de restauración gastronómica del municipio compiten entre sí, ya que varios de ellos comercializan ofertas similares, dirigidas al mismo segmento de mercado.

2-Amenaza de entrada de nuevos competidores: Esta fuerza está siempre latente, por la proliferación de esta actividad como forma de empleo, cada día se insertan más competidores, por un lado, con menores costos, buenas ofertas, precios más bajos, mayor creatividad, estrategias de marketing y publicidad mejor diseñadas. Por otra parte, existe una tendencia a la apertura de establecimientos con propuestas novedosas con estilos más atrevidos, con otros precios, lo que los diferencian de los ya existentes. La aparición de estos nuevos negocios suele ser una mala noticia para los ya establecidos, pero los nuevos concurrentes normalmente aportan no solo una nueva capacidad, sino también recursos significativos e ideas frescas sobre como competir.

3- Poder de negociación con los suministradores: La adquisición de productos y materias primas está distribuida en tres segmentos:

- Tiendas recaudadoras (Red de tiendas Minoristas): Una gran variedad de productos se obtienen de estos establecimientos por lo que en ocasiones se crea un caos ante el déficit de los mismos. Evidenciándose dificultades con la entrada al país de los productos importados y carencia de materia prima para la producción de los productos nacionales, sumándole a esto que son los mismos productos demandados por la población en general, por lo que el suministro es insuficiente.

- Mercados Industriales en CUP: Estos establecimientos también proveen a los hogares ya que ofertan productos a precios más o menos asequibles, pero la demanda supera la oferta, debido a que muchos consumidores provienen del sector privado y acuden a esta opción acaparando grandes cantidades de estos productos para poder minimizar sus costos y optimizar el funcionamiento y rendimiento de sus negocios.

- Ferias y agro-mercado: (estatal-privado): Ambas opciones ofertan productos para todos, pero de igual forma existen deficiencias en cuanto a variedad, cantidad, precio, en ocasiones escasean los productos por temporadas y en el caso de los estatales sufren el infortunio de la transportación y distribución de los productos.

- Ante estas dificultades resultaría factible transferir riesgos hacia las cooperativas agropecuarias, pesqueras, avícolas u otras formas de gestión como alternativa que garantice los suministros con la calidad requerida en tiempo y forma, sobre la base 
de una contratación justa y beneficiosa para ambas partes y prime la relacióncosto-beneficio.

4- Poder negociador del cliente: La principal meta es satisfacer al cliente sobre todas las cosas, por lo que una oferta con precios diferenciados, con políticas de descuentos, pago de comisiones, será una estrategia competitiva para los negocios.

5-Amenaza de productos sustitutos: Para un mejor desarrollo, se renueva periódicamente la carta menú, teniendo en cuenta los productos de temporada, lo que contribuirá a mantener siempre una oferta disponible y variada. Por otra parte, tomar medidas ante la insuficiencia de productos, como la elaboración artesanal de algunos de ellos que minimicen los costos y garanticen la existencia.

Es importante señalar que el éxito de estas empresas estará garantizado si se concentran en conocer las necesidades de los consumidores, además de aunar fuerzas de trabajo y activos también elaboraran estrategias de éxito para entender su entorno competitivo y los factores que determinan la rentabilidad a corto y largo plazo.

\section{Aplicación de la Matriz DAFO}

Este tipo de diagnóstico ayuda a plantear acciones que se deberían poner en marcha para aprovechar las oportunidades detectadas y eliminar o preparar a estos negocios contra las amenazas teniendo conciencia de sus debilidades y fortalezas lo que permitirá definir estrategias, estableciendo objetivos reales y consistentes. Estas estrategias pueden manifestarse de las siguientes maneras: \}

Defensiva: puede adoptarse cuando los negocios están preparados para enfrentarse a las amenazas o si su oferta ya no se considera líder deben resaltar lo que lo diferencia de la competencia. En caso de que disminuya la cuota de mercado, buscar clientes que sean más rentables y protegerlos estableciendo un plan de fidelización de clientes.

Ofensiva: se debe implementar una estrategia de crecimiento cuando las fortalezas son reconocidas por los clientes. Por otra parte, se puede atacar a la competencia para exaltar las ventajas.

Supervivencia: si los negocios se enfrentan a amenazas externas sin las fuerzas internas necesarias para luchar contra la competencia, es inteligente dejar las cosas como están hasta que se asienten los cambios que se produjeron y observar atentamente la internalización del entorno antes de lanzarse a la red. 
Tabla 1: Matriz DAFO.

FORTALEZAS

DEBILIDADES

1. Negocios posicionados por la calidad del producto que ofrecen.

1. Incertidumbre en la captación y selección de mano de obra calificada.

2. Alto sentido de pertenencia de los empleados que contribuyen con su esfuerzo continuo hacer bien las cosas y mejorarlas.

3. Alto grado de reconocimiento por parte de los turistas.

2. Desvinculación del sector turístico.

4. Capital humano, joven, calificado y entusiasta.

5. Exclusividad de la oferta gastronómica.

3. Insuficiente orientación metodológica y capacitación.

4. Difícil acceso a equipamiento tecnológico moderno.

5. Acceso limitado a las NTIC.

6. Mejora de la calidad de vida colectiva e individual de todos los actores involucrados

AMENAZAS

OPORTUNIDADES

1. La competencia constante entre establecimientos que poseen ofertas 1 . Mejoramiento de la imagen del barrio. similares.

2. La escases de productos de todo tipo (insumos, materias primas, productos agrícolas).

3. Ausencia de un mercado mayorista.

4. Marco regulatorio existente para las exportaciones de insumos, materias primas, alimentos.

5. La incidencia de cambios climáticos que afectan la producción de alimentos que aseguran la disponibilidad de materias primas.

6. Inestabilidad en la adquisición de algunos productos en el mercado interno que inciden directamente y de forma negativa en los costos de elaboración.

2. Alineación de los intereses del sector estatal y no estatal.

3. Implementación de las medidas expresadas en los Lineamientos que favorecen al sector no estatal de restauración gastronómica.

4. Estrechar vínculos con otras formas de gestión no estatal

5. Permite al Estado liberarse de algunas prestaciones en relación a los servicios gastronómicos y toda la infraestructura que genera en cuanto a inversiones, equipamiento, suministros, entre otros.

6. Contribuir con el financiamiento del presupuesto para el desarrollo local municipal. 
7. Secuelas que se derivan del deterioro del ambiente urbano.

8. La existencia de destinos gastronómicos en América Latina como: Perú, México, Argentina, Colombia.

9. Falta de respaldo institucional.
7. Posibilidad de diversificación y adquisición de nuevos consumidores.

8. Aumento de la cuota de mercado.

9. Potenciar la competencia entre los establecimientos con ofertas gastronómicas similares.

10. Posibilidad de integración en el sector turístico, no como un complemento de la oferta sino como un producto distintivo.

11. Posicionamiento de Cuba como destino turístico seguro.

12. Crear fuentes de empleos.

Tabla 2: Matriz de Impactos Cruzados

\begin{tabular}{lccccccccccccccccccccccccccc}
\hline & 1 & 2 & 3 & 4 & 5 & 6 & 7 & 8 & 8 & 10 & 11 & 12 & Total & A1 & A2 & A3 & A4 & A5 & A6 & A7 & A8 & A9 & Total & Total \\
\hline F1 & 3 & 3 & 3 & 3 & 3 & 3 & 3 & 3 & 3 & 3 & 3 & 3 & $\mathbf{3 6}$ & 3 & 0 & 0 & 0 & 2 & 2 & 2 & 3 & 2 & $\mathbf{1 4}$ & $\mathbf{5 0}$ \\
F2 & 1 & 2 & 3 & 0 & 3 & 0 & 0 & 0 & 2 & 1 & 0 & 3 & 15 & 0 & 0 & 0 & 0 & 0 & 0 & 0 & 0 & 0 & 0 & $\mathbf{1 5}$ \\
F3 & 3 & 3 & 3 & 3 & 3 & 3 & 3 & 3 & 3 & 3 & 2 & 0 & $\mathbf{3 2}$ & 2 & 0 & 0 & 0 & 2 & 0 & 2 & 3 & 0 & 9 & $\mathbf{4 1}$ \\
F4 & 0 & 3 & 0 & 0 & 0 & 0 & 0 & 0 & 0 & 1 & 0 & 3 & $\mathbf{7}$ & 1 & 0 & 0 & 0 & 0 & 0 & 0 & 0 & 0 & 1 & $\mathbf{8}$ \\
F5 & 3 & 3 & 3 & 3 & 3 & 2 & 3 & 3 & 3 & 3 & 3 & 1 & $\mathbf{3 3}$ & 3 & 3 & 3 & 3 & 3 & 1 & 3 & 3 & 0 & 22 & $\mathbf{5 5}$ \\
F6 & 3 & 3 & 3 & 3 & 0 & 3 & 0 & 0 & 0 & 0 & 0 & 3 & $\mathbf{1 8}$ & 0 & 0 & 0 & 0 & 0 & 0 & 0 & 0 & 0 & 0 & $\mathbf{1 8}$ \\
\hline Total & $\mathbf{1 3}$ & $\mathbf{1 7}$ & $\mathbf{1 5}$ & $\mathbf{1 2}$ & $\mathbf{1 2}$ & $\mathbf{1 1}$ & $\mathbf{9}$ & $\mathbf{9}$ & $\mathbf{1 1}$ & $\mathbf{1 1}$ & $\mathbf{8}$ & $\mathbf{1 3}$ & $\mathbf{1 4 1}$ & $\mathbf{9}$ & 3 & 3 & 3 & 7 & 3 & 7 & 9 & 2 & $\mathbf{4 6}$ & $\mathbf{1 8 7}$ \\
\hline D1 & 0 & 2 & 1 & 1 & 0 & 0 & 0 & 0 & 0 & 0 & 0 & 3 & $\mathbf{7}$ & 0 & 0 & 0 & 0 & 0 & 0 & 0 & 0 & 0 & $\mathbf{3}$ & $\mathbf{1 0}$ \\
D2 & 3 & 3 & 3 & 2 & 3 & 3 & 3 & 3 & 3 & 3 & 3 & 3 & $\mathbf{2 9}$ & 0 & 0 & 0 & 0 & 0 & 0 & 0 & 3 & 3 & $\mathbf{6}$ & $\mathbf{3 5}$ \\
D3 & 0 & 3 & 3 & 0 & 0 & 0 & 0 & 0 & 0 & 0 & 0 & 0 & $\mathbf{6}$ & 0 & 0 & 0 & 0 & 0 & 0 & 0 & 0 & 3 & $\mathbf{3}$ & $\mathbf{9}$ \\
D4 & 0 & 3 & 3 & 0 & 0 & 0 & 3 & 3 & 3 & 3 & 0 & 0 & $\mathbf{1 8}$ & 0 & 0 & 0 & 3 & 0 & 0 & 0 & 1 & 0 & $\mathbf{4}$ & $\mathbf{2 2}$ \\
D5 & 1 & 3 & 3 & 3 & 3 & 0 & 3 & 3 & 3 & 3 & 3 & 0 & $\mathbf{2 8}$ & 3 & 0 & 0 & 0 & 0 & 0 & 0 & 3 & 3 & $\mathbf{9}$ & $\mathbf{3 7}$ \\
\hline Total & $\mathbf{4}$ & $\mathbf{1 4}$ & $\mathbf{1 3}$ & $\mathbf{6}$ & $\mathbf{6}$ & $\mathbf{3}$ & $\mathbf{9}$ & $\mathbf{6}$ & $\mathbf{9}$ & $\mathbf{9}$ & $\mathbf{6}$ & $\mathbf{3}$ & $\mathbf{8 8}$ & $\mathbf{3}$ & 0 & 0 & 3 & 0 & 0 & 0 & 7 & 12 & $\mathbf{2 5}$ & $\mathbf{1 1 3}$ \\
\hline Total & 17 & 31 & 28 & 18 & 18 & 14 & 18 & 15 & 20 & 20 & 14 & 16 & $\mathbf{2 2 9}$ & 12 & 3 & 3 & 6 & 7 & 3 & 7 & $\mathbf{1 6}$ & $\mathbf{1 4}$ & $\mathbf{7 1}$ & $\mathbf{3 0 0}$ \\
\hline
\end{tabular}

Fuente: Elaboración propia a partir de la Matriz DAFO.

El mayor impacto resultante de las interacciones ponderadas se alcanza en el cuadrante de las Fortalezas y Oportunidades. El territorio se encuentra en una posición deseada por lo que deben aprovecharse al máximo las Fortalezas las cuales constituyen sus fuerzas motrices: F1, F2, F3, F5 y F6. Por su parte las Oportunidades que brindan el entorno: O1, O2, O3, O4, O5, O6, O9, O10, O12 tienen condiciones aceptables que potencian los atributos de la localidad para convertirlo en un escenario interesante para el turismo gastronómico, actividad que aportará resultados muy positivos al municipio por lo que se puede desarrollar una estrategia del tipo Ofensiva para una posible implementación. 


\section{Otro enfoque analítico sobre el territorio.}

Con respecto al análisis del macro-entorno donde intervienen un conjunto de condiciones que influyen o afectan de igual manera a todos los negocios y sus dimensiones abarcan diferentes ámbitos:

- Económico: El sector de restauración gastronómica es una sección lucrativa por naturaleza, y la inserción de nuevos espacios genera beneficios para la comunidad y aporta ingresos al presupuesto territorial, en especial al de Centro Habana, por esta razón la apertura de nuevos negocios en la ciudad no escapa de ello, pues la aparición de estos fomenta el desarrollo de la actividad turística urbana.

- Demográfico: Aceptable por la heterogeneidad de la población del municipio, no solo por sus habitantes sino también por la cantidad de personas foráneas temporales que se alojan en los hoteles cercanos y arrendamientos privados, lo que posibilita cierta estabilidad en los consumidores.

- Socio- Cultural: Positivo por el aporte de los diversos estilos de vida de la comunidad, así como sus necesidades y expectativas lo que permite una retroalimentación continúa, intercambio que permite la mejora continua de las ofertas y los servicios.

- Tecnológico: Adecuado, debido a que poseen el equipamiento necesario, tecnología e instalaciones indispensables para el desarrollo de todas las actividades (clima, refrigeración, fluido eléctrico, gas, agua).

- Político- Legal: Respaldado por el decreto Ley Nº141/1993 que legaliza el trabajo por cuenta propia.

- Medio-Ambiental: Ordenado en función del entorno pues deben cumplir con los requisitos de accesibilidad establecidos, sin barreras arquitectónicas. También se aprovechan las condiciones ambientales naturales de luz y aire administrándose los sistemas de climatización e iluminación artificial, así como la reducción de ruidos y olores (compresor de equipo de clima aislado y empleo de extractores para la evitar la concentración del calor y los olores). Es necesario señalar que el municipio presenta un deterioro urbanístico avanzado lo que lacera la imagen local, aunque se han reanimado espacios y viviendas que poseían un alto nivel de deterioro y antigüedad. Existe un alto nivel de colaboración y cordialidad entre los negocios.

Durante el transcurso de la investigación y confección del diagnóstico fue difícil encontrar elementos que permitieran hacer una valoración cuantitativa de los beneficios que genera especialmente este sector a la localidad. Uno de los elementos limitantes fue la desactualización del anuario estadístico del territorio donde se exponen las cifras de todas las actividades económicas y se comparan con los resultados del período anterior lo cual permite el análisis de su comportamiento, cuestión que debería ser importante para la toma de decisiones y formulación de estrategias en función del mejoramiento territorial. Aún así se hace necesario demostrar cuales son las contribuciones de este sector al presupuesto municipal con la intención de expresar el efecto multiplicador que tiene la actividad gastronómica no estatal dentro del municipio. 
Propuesta de acciones que permitan a los negocios no estatales de restauración gastronómica del municipio centro Habana, ser complemento de la oferta extra hotelera.

Una vez concluido el diagnóstico y delimitadas las oportunidades y limitaciones que posee la actividad gastronómica no estatal del territorio esencialmente la de las Paladares se procedió a redactar un conjunto de acciones que posibiliten la integración de las mismas al sector turístico tomando como premisa el impacto que pueden ocasionar en los siguientes aspectos:

Económico: recapacitar con relación al manejo y distribución del Presupuesto Municipal, al cual se destinan estos aportes, aunque queda claro que hay sectores prioritarios (Salud, Educación, Asistencia Social y Deporte) y considerar pate de este a la planificación del presupuesto para el desarrollo local destinado fundamentalmente a la recuperación de los servicios públicos: agua, gas, alumbrado público, viales, recogida de desechos, comunicaciones, mantenimiento constructivo a las viviendas que son de vital importancia para la población, la comunidad y el entorno general contribuyendo a la mejora de la imagen del municipio.

Social: según trabajadores contratados y salarios se garantiza una empleomanía que pudiera ser de mayor incorporación y puede nutrirse de antiguos trabajadores desocupados y calificados, amas de casa, estudiantes y trabajadores estatales con doble vínculo laboral (estatal y no estatal). La elevación del nivel de protección de los trabajadores al ser beneficiados por la Ley de Seguridad Social.

Turismo: existen vías alternativas de apoyo a la red extra-hotelera oferta complementaria a la actividad turística. Con relación a la integración de nuevos conceptos en la gestión turística local se garantiza una nueva forma de desarrollo con diseño de productos integrados permitiendo una estrategia de integración en la gestión de un destino en particular efecto que puede impactar positivamente en el posicionamiento de la marca país, en este caso el destino Cuba.

Desarrollo Local: todos los beneficios soportan, apoyan y garantizan el desarrollo local.

Es interesante comprender los valores que se forjan en los colectivos que en estos establecimientos laboran donde prima: el respeto, la profesionalidad, la cordialidad, el altruismo, la competitividad siendo el capital humano una de sus mayores fortalezas. Aunque es complicado el tema de la capacitación y actualización lo cual queda relegado al trabajo del día a día, a las propias exigencias de la mejora de la calidad continua, el desafío de las escases y las ausencias de determinados elementos que por una parte estimulan la creatividad, la innovación y a veces la improvisación; por otra parte, entorpecen el desarrollo de una actividad tan compleja como la gastronomía. Aún así los establecimientos que hoy existen asumen todas estas dificultades con una tremendísima responsabilidad y luchan por continuar manteniéndose e incluso crecer, pero se hace necesario que exista más respaldo gubernamental que los ampare comprendiendo que su existencia mejora la vida local. 
Las propuestas apuntan hacia:

¿Cómo se puede explotar cada fortaleza?

¿Cómo se puede aprovechar cada oportunidad?

¿Cómo se puede detener cada debilidad?

¿Cómo se puede defender de cada amenaza?

\section{Acciones propuestas}

- Potenciar que los Paladares del municipio Centro Habana, como experiencia, se conviertan en anfitrionas del turismo gastronómico.

- Incentivar el turismo gastronómico como oferta complementaria o como una nueva modalidad turística en la localidad contribuyendo a reforzar la marca país.

- Crear una agencia que promueva e incentive al turismo gastronómico.

- Reforzar los procesos inversionistas en la planta hotelera del municipio en la mejora de la planta habitacional existente y fortalecer los servicios básicos: agua, electricidad, alumbrado público, recogida de desechos, comunicaciones, viales.

- Arrendar al sector no estatal aquellos espacios que las instalaciones hoteleras dispongan y estén aptos para ofrecer servicios gastronómicos o establecer el régimen de cooperativas.

- Fortalecer la promoción de los establecimientos no estatales en las redes sociales, campañas publicitarias de Cuba como destino turístico.

- Apoyar la promoción y publicidad con la ayuda de la NTIC (herramientas on line) para la comunicación del turismo gastronómico.

- Elaborar una guía de establecimientos de restauración gastronómica en diversos soportes, digital, papelería, aplicaciones móviles.

- Diseñar un mapa que contenga la ruta gastronómica de la localidad.

- Fomentar la capacitación y la superación de los profesionales de la gastronomía.

- Divulgar más la existencia de los centros de orientación metodológica con relación a la actividad gastronómica dígase: La Asociación Culinaria y las Escuelas de Hotelería y Turismo.

- Organizar encuentros de comercialización e intercambio.

- Involucrar más a las AAVV en la contratación con las Paladares.

- Actualizar y rediseñar los sitios de Internet cubanos como: Directorio Turístico, La Habana Travel, Cubageotur.

- Permitir el acceso a tecnología avanzada que permita una mejor preparación de las elaboraciones culinarias logrando que estén más a tono con las tendencias actuales.

- Poner en valor los elementos favorables que posee el territorio para el desarrollo de la actividad turística y particularmente en el sector de restauración gastronómica no estatal. 


\section{Conclusiones}

Una vez culminada la investigación se manifiesta que quedaron cumplidos los objetivos propuestos pues se hizo referencia a todos los conceptos empleados. En cuanto al diagnóstico realizado las Paladares del municipio demostraron tener oportunidades y limitaciones para el desarrollo de sus actividades por lo que recomendaron una serie de acciones que pueden incidir en el desarrollo del turismo gastronómico como actividad turística complementaria que favorezca el desarrollo local. De manera concluyente queda resumida de la siguiente manera: No está resuelto en nuestra economía el papel del mercado, que sigue marginado en la práctica.

- El Turismo Gastronómico puede constituir para Cuba en general y sus destinos específicos en particular, una propuesta para la diversificación de sus productos turísticos, tomando como referencia sus atractivos tanto reales como potenciales.

- La conveniencia de la utilización de los Paladares como atractivo turístico local se demuestran porque son capaces de generar empleos para la población residente y sus ganancias se revierten en el desarrollo de la comunidad además de reanimar el espacio urbano.

- Las localidades ostentan valores históricos- culturales y numerosos establecimientos que pueden constituir una motivación para el turismo, además de la idiosincrasia de sus habitantes, elementos que constituyen recursos valiosos y aprovechables para el desarrollo de la actividad turística.

\section{Referencias bibliográficas}

Betancourt, M.E. El turismo, pilar del desarrollo local en Cuba. En www.juventudrebelde.cu/cuba/el-turismo-pilar-del-desarrollo-local/http://. Consultado: 24.10.2014

Guzón, A (2003). Estrategias municipales para el desarrollo. Desarrollo local en Cuba. Retos y perspectivas. La Habana: Editorial Academia.

Dordevic, J. De las tradiciones del gusto y el criollismo del paladar En http://www.cubaliteraria.cu/articulo.php?idseccion=8 - Cubaliteraria/ Consultado: 27.11.2014

Lineamientos de la Política Económica y Social del Partido y la Revolución (2011). En: VI Congreso del Partido Comunista de Cuba. La Habana. Cuba. 
Loyola, V. De lo tradicional y lo Gourmet. En http://www.revistaexcelecias.com/. Consultado: 04.02.2015. 


\section{PARA CITAR EL ARTÍCULO INDEXADO.}

Cisneros Mustelier, L., \& Llanes Rosales, D. (2021). El turismo, pilar del desarrollo local. Los paladares en Cuba. Explorador Digital, 5(1), 64-80. https://doi.org/10.33262/exploradordigital.v5i1.1490

\section{Liencia}

El artículo que se publica es de exclusiva responsabilidad de los autores y no necesariamente reflejan el pensamiento de la Revista Explorador Digital.

El artículo queda en propiedad de la revista y, por tanto, su publicación parcial y/o total en otro medio tiene que ser autorizado por el director de la Revista Explorador Digital.
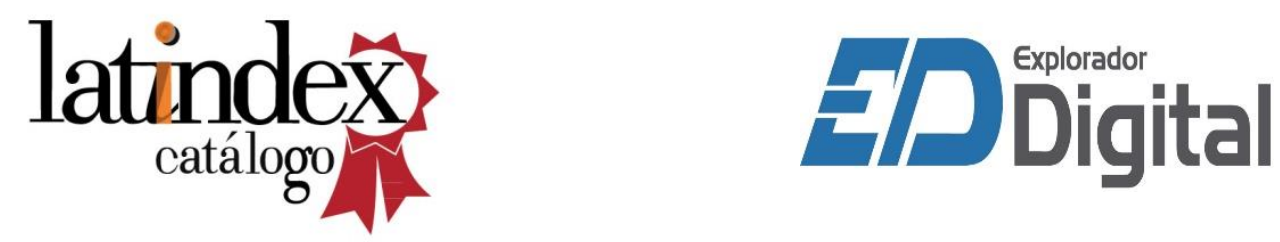